\title{
A METHOD FOR ASTEROID MASS DETERMINATION
}

\author{
M. KUZMANOSKI \\ Faculty of Mathematics \\ Studentski trg 16, 11000 Belgrade, Yugoslavia
}

It is well known that the perturbing effects of any asteroid are negligible at all times, except during a close encounter. The influence of a perturbing asteroid on the motion of a perturbed one can be investigated by means of the differences of the right ascensions and declinations of the perturbed asteroid, as inferred from two numerical integrations, the one with and the other without taking into account the effects of the perturbing asteroid (Kuzmanoski and Knežević, 1994). These effects before reaching the epoch of the close encounter are very small, as expected. Beyond this epoch, the differences increase with time, due to the fact that the orbit is changed on account of the influence of the perturbing body during the close approach, so that perturbations by major planets act on it in a different way.

The essence of this approach is the idea to separate pre-encounter and post-encounter orbits of the perturbed asteroid. In the calculation of these two orbits it is not necessary to know the mass of the perturbing asteroid, because its perturbing effects are practically zero. These two orbits are distinguished by an impulsive change due to the close encounter and can be connected by properly accounting for gravitational effects of the perturbing body. If the pre- and post-encounter orbits are completely precisely determined, the correct mass of perturbing body will give the best fit of the post-encounter observations with the pre-encounter orbit and the preencounter observations with the post-encounter orbit. It is essential that no other close approach (or at least not so close to affect the orbit) occurred in the time spans used for the orbits computation.

With such an approach, we have first calculated pre- and post-encounter orbits and examined how large effects one can expect in right ascensions and declinations for some asteroids which had close encounters with (1) Ceres and have already been used for its mass determination, and found that for (203) Pompeja they were the largest. In the time span covered by observations, two very close encounters occurred between (1) Ceres and 
(203) Pompeja: the closest one (0.017 AU) in August 1948, and the second one $(0.08 \mathrm{AU})$ in February 1969, while a few more approaches occurred within $0.5 \mathrm{AU}$. Next, two orbits were calculated: the first one by using the observations from the time span up to the epoch of the close encounter (41 observations at 19 oppositions and RMS residual $2^{\prime \prime} .01$ ), and the other one by using the ones from the time span after the second close approach $\left(48,12,1^{\prime \prime} \cdot 26\right)$. Numerical integrations were made by means of a Radau integrator of order 15, developed by Everhart (1985).

With the pre-encounter orbit we have fitted post-encounter observations (in turn used in calculation of the post-encounter orbit), and by means of the post-encounter orbit the pre-encounter observations (used in calculation of the pre-encounter orbit), setting the trial values for the mass of (1) Ceres and computing $\mathrm{O}-\mathrm{C}$ residuals in the RMS sense. Obtained results are shown below:

TABLE 1. RMS residuals of post-encounter (a) and pre-encounter (b) observations for different trial masses $(\mathrm{m})$

\begin{tabular}{lllllllllll}
\hline & $1 / \mathrm{m}\left(10^{9}\right)$ & 2.00 & 2.10 & 2.20 & 2.30 & 2.40 & 2.50 & 2.60 & 2.70 & $\mathrm{~m}=0$ \\
\hline a) & RMS (") & 6.70 & 4.89 & 3.31 & 2.03 & 1.45 & 1.95 & 2.90 & 3.91 & 34.09 \\
b) & RMS (") & 7.75 & 5.21 & 3.19 & 2.33 & 3.19 & 4.71 & 6.31 & 7.85 & 50.22 \\
\hline
\end{tabular}

As can be seen, the best representation of post-encounter observations has been obtained for $1 / m=2.4 \cdot 10^{9}$ ( RMS residual $1^{\prime \prime} 45$ ), and of preencounter observations for $1 / m=2.3 \cdot 10^{9}$ (RMS residual $2^{\prime \prime} \cdot 33$ ). This preliminary result is not in agreement with those derived by Goffin (1991) and Bowell et al. (1994) for the same close encounter. The difference could be to the inaccuracy of initial orbits, neglecting of the other close encounters influence, different integrators used, but also to the different methods for mass determination.

\section{References}

Bowell E., Muinonen K., Wasserman L. H. (1994) Asteroid mass determination from multiple asteroid-asteroid encounters, Proceedings of Mariehamn, in press.

Everhart, E. (1985) An efficient integrator that uses Gauss-Radau spacings, in Carusi A., Valsecchi G.B. eds, Dynamics of Comets: Their Origin and Evolution, 185-211

Goffin E. (1991) The orbit of 203 Pompeja and the mass of Ceres, Astron. Astrophys., 249, 563-568

Kuzmanoski M., Knežević Z. (1994) Asteroid close encounters and mutual perturbations, Planet. Space Sci., 42, 297-299 